\title{
Scale interactions and scaling laws in rotating flows at moderate Rossby numbers and large Reynolds numbers
}

\author{
P. D. Mininni, ${ }^{1,2}$ A. Alexakis, ${ }^{3}$ and A. Pouquet $^{2}$ \\ ${ }^{1}$ Departamento de Física, Facultad de Ciencias Exactas y Naturales, Universidad de Buenos Aires, \\ Ciudad Universitaria, 1428 Buenos Aires, Argentina \\ ${ }^{2}$ NCAR, P.O. Box 3000, Boulder, Colorado 80307-3000, USA \\ ${ }^{3}$ Laboratoire Cassiopée, Observatoire de la Côte d'Azur, BP 4229, Nice Cedex 04, France
}

(Received 25 February 2008; accepted 4 November 2008; published online 21 January 2009)

\begin{abstract}
The effect of rotation is considered to become important when the Rossby number is sufficiently small, as is the case in many geophysical and astrophysical flows. Here we present direct numerical simulations to study the effect of rotation in flows with moderate Rossby numbers (down to Ro $\approx 0.03$ ) but at Reynolds numbers large enough to observe the beginning of a turbulent scaling at scales smaller than the energy injection scale. We use coherent forcing at intermediate scales, leaving enough room in the spectral space for an inverse cascade of energy to also develop. We analyze the spectral behavior of the simulations, the shell-to-shell energy transfer, scaling laws and intermittency, as well as the geometry and the anisotropy of the structures in the flow. At late times, the direct transfer of energy at small scales is mediated by interactions with the largest scale in the system, the energy containing eddies with $k_{\perp} \approx 1$, where $\perp$ refers to wavevectors perpendicular the axis of rotation. The transfer between modes with wavevector parallel to the rotation is strongly quenched. The inverse cascade of energy at scales larger than the energy injection scale is nonlocal, and energy is transferred directly from small scales to the largest available scale. We observe both a direct and inverse cascade of energy at high rotation rate, indicative that these cascades can take place simultaneously. Also, as time evolves and the energy piles up at the large scales, the intermittency of the direct cascade of energy is preserved while corrections due to intermittency are found to be the same (within error bars) as in homogeneous nonrotating turbulence. () 2009 American Institute of Physics. [DOI: 10.1063/1.3064122]
\end{abstract}

\section{INTRODUCTION}

Strong rotation is present in many geophysical and astrophysical flows. Its effect is considered to become important when the Rossby number (the ratio of the convective to the Coriolis acceleration, or the ratio of the rotation period to the eddy turn-over time) is sufficiently small. The large scales of atmospheric and oceanic flows, for example, are affected by the rotation of the Earth. The Rossby number for midlatitude synoptic scales in the atmosphere is $\mathrm{Ro} \approx 0.1{ }^{1}$ In the Sun, the typical Rossby number in the convective zone is Ro $\approx 0.1-1$ (Ref. 2). Furthermore, the Reynolds number (Re, the ratio of the convective to the viscous acceleration) in these systems is also very large, and the flows are in a turbulent state.

Many studies have considered the effect of rotation in a turbulent flow, as a first step to gain better understanding of the fluid dynamics of geophysical systems, for which, e.g., stratification also plays an important role. For rapid rotation (very small Rossby numbers), significant progress has been made by applying resonant wave theory, ${ }^{3,4}$ two-point spectral closures, ${ }^{5,6}$ and weak turbulence theory. ${ }^{7}$ In these approaches, the flow is considered as a superposition of inertial waves with a short period, and the evolution of the system for long times is derived considering the effect of resonant triad interactions.

Recently, resonant wave theory has been shown to correspond to an asymptotic limit for a general class of geo- physical flows with wave dynamics. ${ }^{8,9}$ Also, the connection between resonant wave theory and two-point closures based on helical modes ${ }^{4,5}$ was shown in Ref. 10. This approach sheds light on the mechanism that drives the flow to be quasi-two dimensional (quasi-2D) at large scales: ${ }^{4,5}$ energy in three-dimensional modes is transferred by a subset of the resonant interactions to modes with smaller vertical wavenumber. It also explains successfully the observed enhanced transfer of energy from the small to the large scales. ${ }^{11}$

However, it is well known that wave theory is only valid when the wave period (the rotation period here) is much shorter than the eddy turn-over time at all scales. For large Reynolds numbers, small scales are excited with a characteristic timescale proportional to the eddy turn-over time that decreases as the scales become smaller. Therefore the approximations made in such theories can break down at sufficiently small scales, provided that the Reynolds number is large enough for these scales to be excited. How the results of resonant wave theory extend to the case of only moderate Rossby numbers but very large Reynolds numbers is still unclear. Two-point spectral closures ${ }^{5,6}$ can be used to match the two regimes using the same theoretical tool (see, e.g., Ref. 10). This shows that the energy transfer is anisotropic even at moderate Rossby numbers because of linear phase mixing, and that resonant triads are selected in the limit of small Rossby number (see also Refs. 12 and 13).

Another approach for understanding rotating flows at 
large Reynolds numbers is to develop phenomenological models based on scaling arguments and self-similarity. Initial phenomenological investigations considered isotropic energy spectra $E(k)$ (Refs. 14 and 15) that suggested the power law behavior $E(k) \sim k^{-2}$ provided that the examined wavenumbers were smaller than a critical wave number $k_{\Omega} \sim \sqrt{\Omega^{3} / \epsilon}$, where $\epsilon$ is the energy dissipation rate. For larger wavenumbers, the energy spectrum transitioned to the isotropic Kolmogorov energy spectrum $E(k) \sim k^{-5 / 3}$. Anisotropy was taken into account in phenomenological models in Ref. 16, where it was recognized that a considerable amount of the energy will be confined in "quasi-2D" modes. An anisotropic energy spectrum $E(k) \sim k_{\perp}^{-2}$ (where $k_{\perp}$ denotes the wavevectors perpendicular to $\boldsymbol{\Omega}$ ) with a non-self-similar behavior along the axis parallel to the rotation has been suggested $i^{17}$ on the basis of numerical results.

In numerical simulations, the study of rotating turbulent flows is constrained by the computational cost of properly resolving the inertial waves and the resonant triadic interactions, together with the cost of resolving the small-scale fluctuations when the Reynolds number is large. Inverse cascades were shown to develop and anisotropies to appear in low resolution $\left(32^{3}\right.$ and $64^{3}$ grid points) simulations, ${ }^{18-20}$ either solving the equations of motion directly or using a subgrid model. Small aspect ratio boxes were considered in Refs. 21 and 22 allowing for an increase in resolution. Simulations at higher resolution were done later in Ref. 23 studying, in particular, the behavior of the shell-to-shell energy transfer. Recently, simulations with large Reynolds number and small Rossby number were performed using $128^{3}$ grids and eighth-order hyperviscosity, ${ }^{24}$ thus confirming the dominant role of resonant triads for rapid rotation at large Re, although the results also suggest that resonant wave theory can be valid only for a finite interval of time; this has been argued by a number of authors in the general context of wave turbulence because of the nonuniformity in scale of the method, as already discussed earlier. Also note that all these simulations give different results for the scaling of the energy spectrum at scales larger than the forcing scale; it was shown in Ref. 11, using a truncated model that this can be the result of how all the relevant time scales are resolved. A synthesis of dynamical and anisotropic aspects of rotating turbulence, with discussion of numerical and physical experiments and models, can be found in Ref. 25 .

In this paper, we study the effect of rotation in a turbulent flow using high resolution direct numerical simulations with up to $512^{3}$ grid points. Simulations at this resolution were also performed recently in Ref. 17 with an injection of energy at the largest scale available; the focus was solely on the scaling of small-scale fluctuations, showing depletion of the energy cascade and reduced intermittency. Our main objective, on the other hand, is to study the statistical properties of the fluctuations in flows with moderate Rossby numbers (down to $\mathrm{Ro} \approx 0.03$ ) but at Reynolds numbers large enough to observe the beginning of a turbulent scaling at scales larger and smaller than the energy injection scale.

To this end, we use coherent forcing at intermediate scales, leaving enough room in the spectral space for an inverse cascade of energy to develop when the Rossby number is small enough. We also use the largest value of the Reynolds number allowed by our grid to observe a direct transfer of energy at small scales. After describing the simulations, we study the spectral behavior of the energy in the runs, the shell-to-shell energy transfer, scaling laws and intermittency, the geometry of the structures, and finally the degree of anisotropy of the resulting flow.

\section{NUMERICAL SIMULATIONS}

We solve numerically the equations for an incompressible rotating fluid with constant mass density,

$$
\frac{\partial \mathbf{u}}{\partial t}+\boldsymbol{\omega} \times \mathbf{u}+2 \boldsymbol{\Omega} \times \mathbf{u}=-\nabla \mathcal{P}+\nu \nabla^{2} \mathbf{u}+\mathbf{F}
$$

and

$$
\nabla \cdot \mathbf{u}=0,
$$

where $\mathbf{u}$ is the velocity field, $\boldsymbol{\omega}=\nabla \times \mathbf{u}$ is the vorticity, $\mathcal{P}$ is the total pressure (modified by the centrifugal term) divided by the mass density, and $\nu$ is the kinematic viscosity. Here, $\mathbf{F}$ is an external force that drives the turbulence, and we chose the rotation axis to be in the $z$ direction: $\boldsymbol{\Omega}=\Omega \hat{z}$, with $\Omega$ the rotation frequency.

The mechanical forcing $\mathbf{F}$ is given by the Taylor-Green (TG) flow $^{26}$

$$
\begin{aligned}
\mathbf{F}= & F_{0}\left[\sin \left(k_{0} x\right) \cos \left(k_{0} y\right) \cos \left(k_{0} z\right) \hat{x}\right. \\
& \left.-\cos \left(k_{0} x\right) \sin \left(k_{0} y\right) \cos \left(k_{0} z\right) \hat{y}\right],
\end{aligned}
$$

where $F_{0}$ is the forcing amplitude. The forcing injects energy directly only into the $x$ and $y$ components of the velocity, but with a three-dimensional dependence. However, in the absence of rotation $(\Omega=0)$, the resulting flow is fully three dimensional even in the absence of instabilities (turbulence) because pressure gradients excite the $z$ component of the velocity. ${ }^{26,27}$ The TG flow has a small spectral anisotropy with slightly more energy in the $z$ direction (see Sec. VI for more details), an effect that is the opposite of the tendency toward two dimensionalization due to rotation when $\Omega$ is nonzero. The flow has no net helicity, although locally regions with strong positive and negative helicity develop. It is also worth noting that this forcing injects zero energy in the $k_{z}=0$ mode, whose amplification observed in the strongly rotating cases (see below) is thus only due to a cascade process.

The TG flow is important in hydrodynamics for the insights it provides. It was originally motivated as an initial condition which, though highly symmetric, would lead to the rapid development of small spatial scales, ${ }^{26}$ and as a result proposed as a paradigm of the direct cascade of energy in turbulence. It also mimics the von Kármán flows between two counter-rotating disks used in several turbulence experiments, including experiments of rotating turbulence ${ }^{28}$ and recent experiments to reproduce generation of magnetic fields by dynamo action. ${ }^{29}$

Two sets of runs were done at resolutions of $256^{3}$ (set A) and $512^{3}$ grid points (set B). The parameters for all the runs are listed in Table I. With TG forcing, the spherical shell in Fourier space where energy is injected has wavenumber $k_{F}$ 
TABLE I. Parameters used in the simulations. $N$ is the linear grid resolution, $k_{0}$ the wavenumber used in the forcing, $\nu$ the kinematic viscosity, $\Omega$ the rotation rate, $t_{\max }$ the maximum number of turn-over times computed; $\mathrm{Re}, \mathrm{Ro}$, and Ek are, respectively, the Reynolds, Rossby, and Ekman numbers. Note that runs A and B1 all start from a fluid at rest, whereas runs B2 and B3 start from the steady state reached by run B1 (see text).

\begin{tabular}{|c|c|c|c|c|c|c|c|c|}
\hline Run & $N$ & $k_{0}$ & $\nu$ & $\Omega$ & $t_{\max }$ & $\operatorname{Re}$ & Ro & Ek \\
\hline A1 & 256 & 2 & $2 \times 10^{-3}$ & 0.08 & 45 & 900 & 4.50 & $5 \times 10^{-3}$ \\
\hline $\mathrm{A} 2$ & 256 & 2 & $2 \times 10^{-3}$ & 0.40 & 45 & 900 & 0.70 & $8 \times 10^{-4}$ \\
\hline $\mathrm{A} 3$ & 256 & 2 & $2 \times 10^{-3}$ & 0.80 & 45 & 900 & 0.35 & $4 \times 10^{-4}$ \\
\hline $\mathrm{A} 4$ & 256 & 2 & $2 \times 10^{-3}$ & 1.60 & 45 & 900 & 0.17 & $2 \times 10^{-4}$ \\
\hline A5 & 256 & 2 & $2 \times 10^{-3}$ & 3.20 & 150 & 900 & 0.09 & $1 \times 10^{-4}$ \\
\hline A6 & 256 & 2 & $2 \times 10^{-3}$ & 8.00 & 185 & 900 & 0.03 & $3 \times 10^{-5}$ \\
\hline B1 & 512 & 4 & $8 \times 10^{-4}$ & 0.40 & 17 & 1100 & 1.40 & $1 \times 10^{-3}$ \\
\hline B2 & 512 & 4 & $8 \times 10^{-4}$ & 1.60 & 25 & 1100 & 0.35 & $3 \times 10^{-4}$ \\
\hline B3 & 512 & 4 & $8 \times 10^{-4}$ & 8.00 & 40 & 1100 & 0.07 & $6 \times 10^{-5}$ \\
\hline
\end{tabular}

$=\sqrt{3} k_{0}$, or equivalently, at a scale $L_{F}=2 \pi / k_{F}$. For the runs in set $\mathrm{A}, k_{F} \approx 3.5$, and for the runs in set $\mathrm{B}, k_{F} \approx 6.9$; as a result, there is more room in spectral space for an inverse cascade to take place in the B runs; the B runs also have a slightly higher Reynolds number.

All the runs in set A were started from a fluid at rest. At $t=0$, the rotation and the external forcing were switched on, until reaching a turbulent steady state, or until an inverse cascade was well developed in the case of large rotation rates. The runs in set B were done as follows. Run B1 was started from a fluid at rest and after turning on the rotation and external forcing, the run was continued to reach a turbulent steady state. Runs B2 and B3 were started from a snapshot of the velocity field from the steady state of run B1, and both runs were continued until a new steady state was reached, or an inverse cascade developed. This latter method proved useful in saving computing time, as no differences were observed when comparing the late time evolution of the runs in the two sets. However, both methods were used in order to measure the extent of the transient regime in each case (see Sec. III), and make sure the energy transfer and other relevant quantities were measured in the developed turbulent regime. To this end, all simulations were extended for very long times. Note that times in Table I and in the figures are expressed in units of the turn-over time at the forcing scale. Finally, in all simulations, a dissipative range was properly resolved, and the time step was much smaller than all the relevant timescales.

We define the integral and Taylor scales of the flow respectively as

$$
L=2 \pi \frac{\int E(k) k^{-1} d k}{\int E(k) d k}
$$

and

$$
\lambda=2 \pi\left(\frac{\int E(k) d k}{\int E(k) k^{2} d k}\right)^{1 / 2},
$$

where $E(k)$ is the energy spectrum. Since for large $\Omega$ an inverse cascade develops, these two scales are useful to describe the evolution of characteristic scales in the flow with time. However, to avoid a time dependence of the Reynolds and Rossby numbers (time dependent Rossby numbers based on inertial range scales are discussed in Secs. V and VI), we define for each run the Reynolds number as

$$
\operatorname{Re}=\frac{L_{F} U}{\nu},
$$

and the Rossby number as

$$
\mathrm{Ro}=\frac{U}{2 \Omega L_{F}} .
$$

We also define the Ekman number as

$$
\mathrm{Ek}=\frac{\mathrm{Ro}}{\operatorname{Re}}=\frac{\nu}{2 \Omega L_{F}^{2}} .
$$

Note that other Rossby, Reynolds, and Ekman numbers can be defined based on different choices for the characteristic length scales (e.g., the integral and Taylor scales, or the anisotropic integral scales based on the parallel and perpendicular energy spectra discussed in Sec. III). For simplicity, in the following when we refer to Reynolds, Rossby, or Ekman numbers, the definitions based on the forcing scale $L_{F}$ are to be assumed except stated otherwise. Rossby numbers based on small-scale quantities such as the Taylor scale or the rms vorticity (discussed mostly in Sec. V) are referred to as micro-Rossby numbers.

Based on these definitions, the turn-over time at the forcing scale is then defined as $T=L_{F} / U$, where $U=\sqrt{\left\langle u^{2}\right\rangle}$ is the rms velocity measured in the turbulent steady state, or when the inverse cascade starts. The amplitude of the forcing $F_{0}$ in the simulations is increased as $\Omega$ is increased in order to have $U \approx 1$ in all the runs.

\section{TIME EVOLUTION AND SPECTRA}

Figure 1 shows the time history of the energy in the runs in set A. Runs A1-A4 show a similar evolution, but runs A5 and A6 evolve differently. As the Rossby number decreases, a transient develops in which the total energy oscillates with a frequency that increases with $\Omega$. Examining the inset, the period of oscillation of the total energy in that first phase decreases monotonically from run $\mathrm{A} 3$ to run $\mathrm{A} 6$; for the dis- 


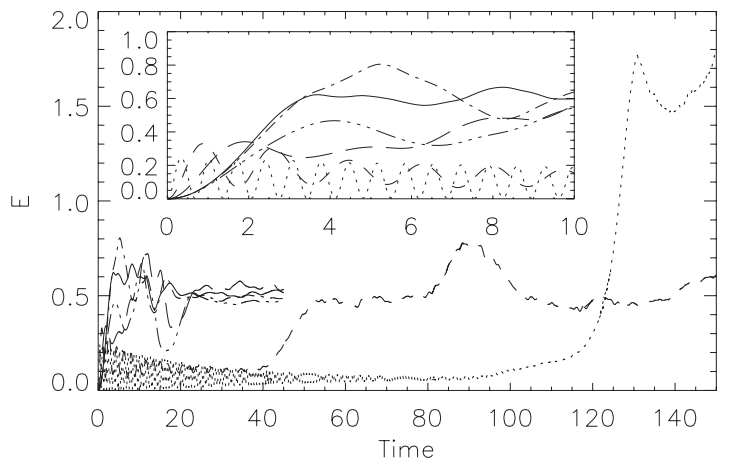

FIG. 1. Time history of the energy for set A: A1 (solid), A2 (dashed-dotted), A3 (dashed-triple dot), A4 (long dashed), A5 (dashed), and A6 (dotted); Rossby numbers range from 4.50 to 0.03 . The inset shows a detail of the evolution at early times: At high rotation rates (runs A5 and A6), waves prevail and the energy of the system is lower than when little rotation is present. Note the transition to a high energy regime at later times as Ro decreases, because of an inverse energy cascade setting in (run A6 continues until $t \approx 185$, while the energy keeps growing steadily).

sipation (see below) this oscillation is only clearly seen at the lowest Rossby number in run A6. The transition time between this transient regime to another regime grows as $\Omega$ increases, for sufficiently large $\Omega$. Then, the energy increases suddenly and a fully turbulent regime finally develops, including at the lowest Rossby number. An inverse cascade of energy is observed in run A6 after $t \approx 120$. The increase in the energy observed after this time is also accompanied by a monotonous increase with time of the flow integral scale $L$. Even in the runs in set B that are restarted from a preexisting turbulent steady state, long runs are needed to reach another turbulent state after turning on the rotation. As an example, in run $\mathrm{B} 3$ it takes $\approx 20$ turn-over times for the transient to decay and for an inverse cascade of energy to develop.

The energy dissipation rate $2 \nu \Omega_{\nu} \equiv \nu \int \omega^{2} d V$ as a function of time is shown in Fig. 2 As the Rossby number decreases, the peak of the dissipation rate is reached at later times, and then it saturates. Note that during the early transient in runs A5 and A6, the dissipation is almost negligible, while in the saturated state the mean dissipation rate decreases slowly with decreasing Rossby number.

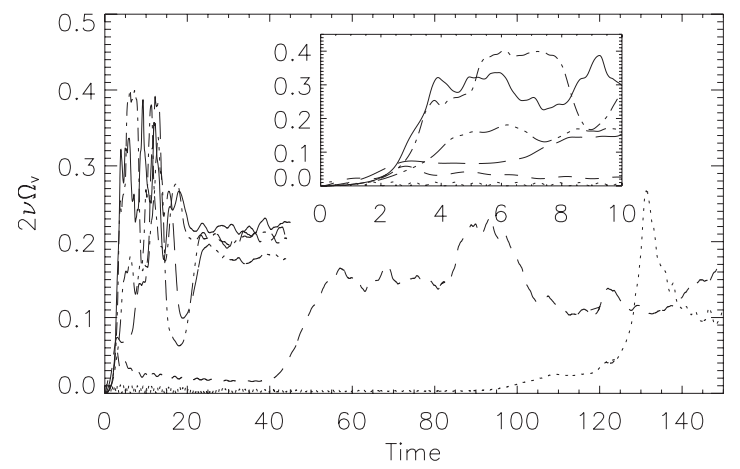

FIG. 2. Time history of the energy dissipation rate (labels as in Fig. 1); the inset again shows the evolution at early times. Note that after transients with little dissipation, runs A3 to A6 reach approximately half the level of turbulence activity of the nonrotating flows, as measured by their dissipation rate.

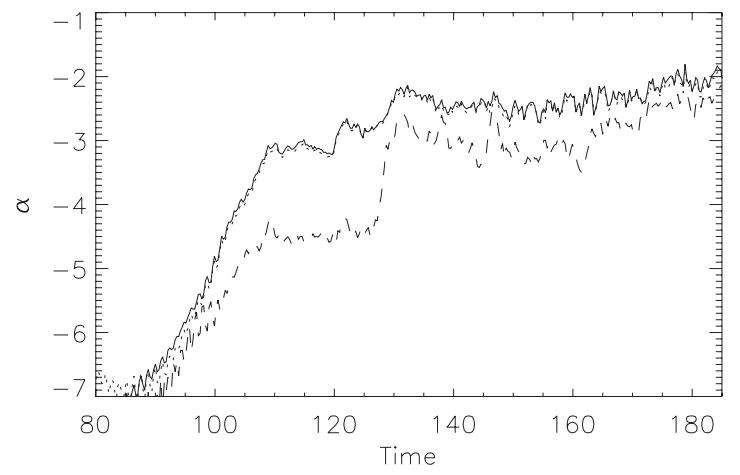

FIG. 3. Spectral index $\alpha$ as a function of time in run A6, in the isotropic energy spectrum $E(k)$ (solid), in the $E\left(k_{\perp}\right)$ spectrum (dotted), and in the $E\left(k_{\|}\right)$spectrum (dashed). Note that the energy distribution, as measured by $\alpha$, is dominated by the perpendicular modes.

The shape of the energy spectrum evolves with time, specially after the transient as turbulence sets in, and later again as the spectrum becomes dominated by the contribution from the largest scales when the Rossby number is small enough for an inverse cascade to develop. Figure 3 shows the time evolution of the spectral index $\alpha$ (the exponent in the region of the spectrum with $k>k_{F}$ that follows a power law $\sim k^{\alpha}$ ) in run A6. Three curves are shown, which correspond respectively to the spectral index computed on the isotropic energy spectrum $E(k)$, on the perpendicular energy spectrum $E\left(k_{\perp}\right)$ (where $k_{\perp}$ denotes the wavevectors perpendicular to $\boldsymbol{\Omega}$ ), and the parallel spectrum $E\left(k_{\|}\right)$(where $k_{\|}$denotes the wavevectors parallel to $\boldsymbol{\Omega}$ ). Here and in the following, the isotropic energy spectrum $E(k)$ is defined by averaging in Fourier space over spherical shells, and the reduced energy spectra $E\left(k_{\perp}\right)$ and $E\left(k_{\|}\right)$are defined averaging in Fourier space respectively over cylinders and over planes; a detailed definition of these averages can be found in Sec. IV, Eqs. (10)-(12).

Before $t \approx 80$, we cannot recognize a power law in the energy spectra. After $t \approx 80$, the spectral indices in $E(k)$ and $E\left(k_{\perp}\right)$ grow monotonically from a value of -7 until reaching a plateau with $\alpha \approx-3$ at $t \approx 110$. The energy spectra $E(k)$, $E\left(k_{\perp}\right)$, and $E\left(k_{\|}\right)$show wide and steep power law behavior from $t \approx 80$ to $t \approx 120$. During this transient, the energy flux is almost zero, as can also be expected from the small value of the energy dissipation in run A6 before $t \approx 110$ (Fig. 2). The end of the transient at $t \approx 110$ and the plateau in $\alpha$ correspond, respectively, to the increase in the energy and in the energy dissipation rate showed in Figs. 1 and 2 . The spectral index in $E\left(k_{\|}\right)$also has a plateau with $\alpha \approx-4.5$. However, as the inverse cascade sets in and the energy piles up at the largest available scale in the system, the spectral index changes again and seem to slowly evolve toward $\alpha \approx-2$ in both $E(k)$ and $E\left(k_{\perp}\right)$. $E\left(k_{\|}\right)$evolves toward a steeper spectrum with a strong peak at $k_{\|}=0$.

Note that the inverse cascade only starts after $\approx 10$ turnover times after the turbulent state is reached at $t \approx 110$. This can be understood as follows. The energy spectrum observed before $t \approx 110$ has almost no flux. Nonlinear transfer of energy is required for the flow to become $2 \mathrm{D}$ under the effect of rotation, $4,6,22$ and the nonlinear transfer is negligible until 


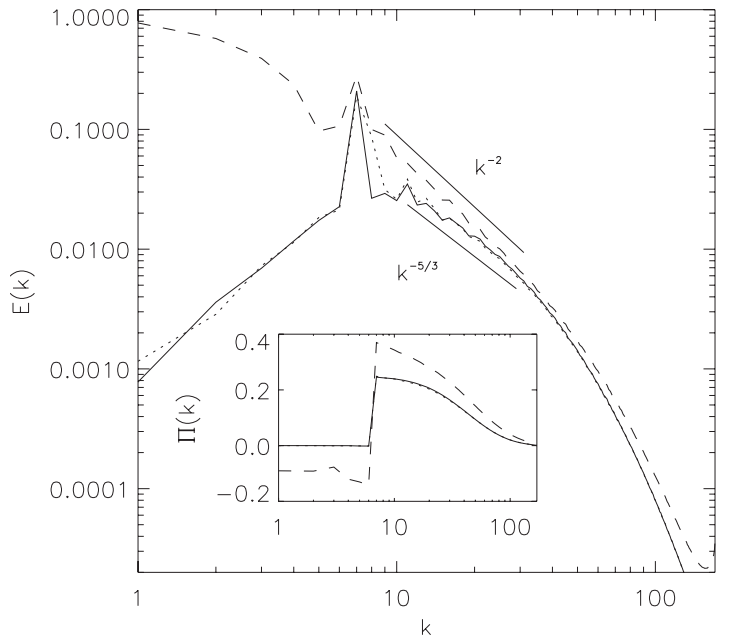

FIG. 4. Isotropic energy spectra at late times in runs B1 (solid, $t \approx 16$ ), B2 (dotted, $t \approx 24$ ), and B3 (dashed, $t \approx 40$ ) at low Ro. Two slopes are given as a reference. The inset shows the isotropic energy flux for the same runs.

$t \approx 110$. Then, after a few turn-over times, the flow undergoes a transition and the inverse cascade sets in.

The long transient is only observed in the runs in set A since the runs in set B are started from a turbulent steady state. However, after the transient, the spectral evolution of the runs in sets A and B is similar. Since runs in set B have more scale separation for an inverse cascade to develop when Ro is small enough, we focus now on this set of runs. We show in Fig. 4 the isotropic energy spectrum at late times in runs B1-B3. While runs B1 and B2 show no growth of energy at scales larger than the mechanical forcing, except for some backscattering with an $\sim k^{2}$ spectrum, run B3 at late times is dominated by the energy in the $k=1$ shell. At scales smaller than the forcing scale, the spectrum of run B3 is steeper than that of runs B1 and B2, and compatible with an $\sim k^{-2}$ scaling. The inset in Fig. 4 shows the isotropic energy flux in the same runs. Note that in run B3, the flux at scales larger than the forcing scale is negative and approximately constant, indicating the development of an inverse cascade of energy for small Ro, although at a lower intensity than the direct cascade. At smaller scales, the energy flux is positive. We thus conclude that in rotating flows, both the direct and inverse energy cascades can cohabit.

The energy spectrum $E\left(k_{\perp}\right)$ is shown in Fig. 5, together with the energy flux $\Pi\left(k_{\perp}\right)$. The spectrum and flux are similar to the isotropic ones (indicating that most of the energy is in these modes), and $\Pi\left(k_{\perp}\right)$ confirms the development of an inverse cascade of energy in $k_{\perp}$ at scales larger than the forcing scale in run B3, and a direct cascade at smaller scales with an $\sim k_{\perp}^{-2}$ scaling. Figure 6 shows the energy flux $\Pi\left(k_{\perp}\right)$ normalized by the rms velocity in each run. Note that the increase of the flux observed in the inset of Fig. 5 is only due to the increase in the energy of the system as the inverse cascade piles up energy at the largest available scale. As Fig. 6 indicates, the actual transfer of energy is slowed down by the rotation, and run B3 shows a smaller normalized flux than the other two runs at scales smaller than the forcing scale.

On the other hand, there is no clear scaling in the small

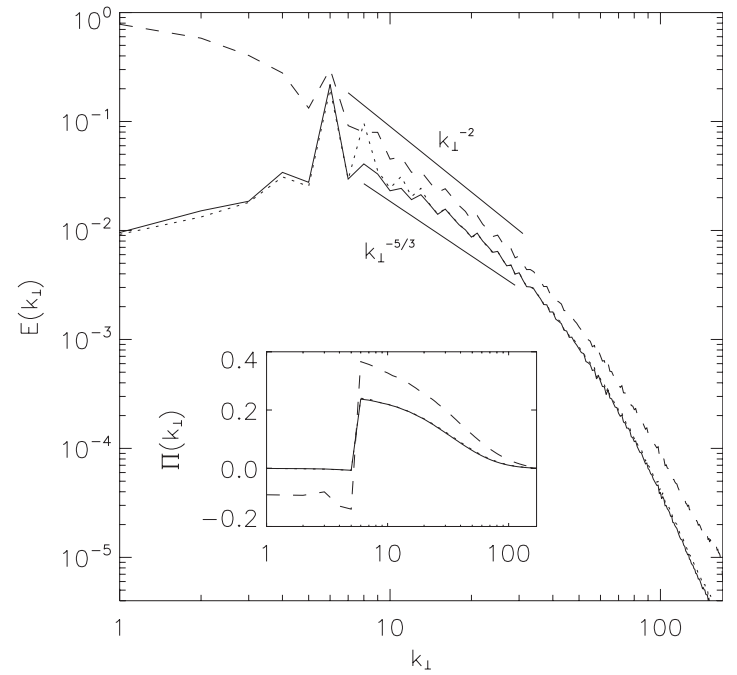

FIG. 5. $E\left(k_{\perp}\right)$ and $\Pi\left(k_{\perp}\right)$ (inset) at late times in runs B1, B2, and B3. Labels are as in Fig. 4.

scales in $E\left(k_{\|}\right)$, nor an inverse cascade at large scales (see Fig. 7). The $E\left(k_{\|}\right)$spectrum in run B3 is steeper than the $E\left(k_{\perp}\right)$ spectrum, consistent with the results shown in Fig. 3 for run A6 at late times. Slopes $\sim k^{-5 / 3}$ and $\sim k^{-2}$ are shown in Fig. 7 only as a reference.

Before proceeding to the computation of the transfer function and other high order statistics, it is worth pointing out that the long transient observed in runs in set A, as well as a shorter transient observed at early times in the runs in set $\mathrm{B}$, make computation of long runs crucial for the following analysis. In run A6, which was started from a fluid initially at rest, the inverse cascade only starts after $t \approx 120$. However even in run B3, which was started from a previous turbulent steady state, a transient with oscillations in the energy and the enstrophy is observed at early times, which are damped only after $\approx 10$ turn-over times.

\section{ENERGY TRANSFER}

In this section we study the scale interactions and energy transfer in rotating turbulent flows. A study of the energy transfer in this context, although at lower resolution and earlier times, was done before by Yeung and Zhou. ${ }^{23}$ We will focus on runs B1, B2, and B3 that have enough scale sepa-

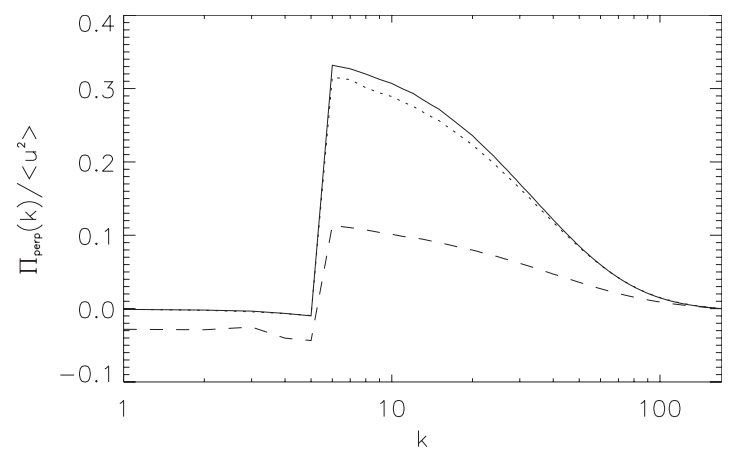

FIG. 6. $\Pi\left(k_{\perp}\right) /\left\langle u^{2}\right\rangle$ at late times in runs B1, B2, and B3. Labels are as in Fig. 4. 


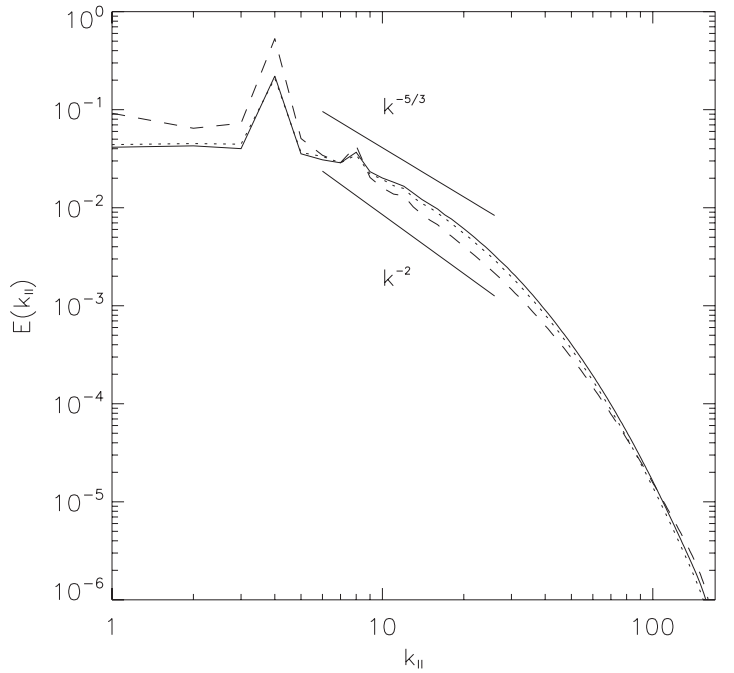

FIG. 7. $E\left(k_{\|}\right)$at late times in runs B1, B2, and B3. Labels are as in Fig. 4. In addition, there is a strong peak at $k_{\|}=0$ for run $\mathrm{B} 3$, of amplitude $E\left(k_{\|}\right.$ $=0) \approx 2$, that cannot be seen in the figure due to the logarithmic scaling used.

ration for direct and inverse cascades to develop when Ro is small enough. Similar results were obtained in the analysis of the runs in set A.

To investigate the transfer of energy among different scales we consider the shell filter decomposition of the velocity field,

$$
\mathbf{u}(\mathbf{x})=\sum_{K} \widetilde{\mathbf{u}}_{K}(\mathbf{x}),
$$

where $K$ denotes a foliation of Fourier space in shells that for our purposes can be taken as spheres ${ }^{30-33}$

$$
\mathbf{u}_{K}(\mathbf{x})=\sum_{K \leq|\mathbf{k}| \leq K+1} \widetilde{\mathbf{u}}_{\mathbf{k}} e^{i \mathbf{k} \cdot \mathbf{x}},
$$

cylinders $^{34}$

$$
\mathbf{u}_{K_{\perp}}(\mathbf{x})=\sum_{K \leq\left|\mathbf{\mathbf { k } _ { \perp }}\right| \leq K+1} \widetilde{\mathbf{u}}_{\mathbf{k}} e^{i \mathbf{k} \cdot \mathbf{x}},
$$

or planes ${ }^{34}$

$$
\mathbf{u}_{K_{\|}}(\mathbf{x})=\sum_{K \leq\left|\mathbf{k}_{\|}\right| \leq K+1} \widetilde{\mathbf{u}}_{\mathbf{k}} e^{i \mathbf{k} \cdot \mathbf{x}} .
$$

Then, we can define the shell-to-shell transfer between these shells as

$$
T(Q, K)=-\int \mathbf{u}_{\mathbf{K}}(\mathbf{u} \cdot \nabla) \mathbf{u}_{\mathbf{Q}} d \mathbf{x}^{3} .
$$

This function expresses the transfer rate of energy lying in the shell $Q$ to energy lying in the shell $K$. It satisfies the symmetry property $T(Q, K)=-T(K, Q),{ }^{30}$ and the numbers labeling the shells $Q$ and $K$ can correspond to any of the foliations of Fourier space listed above. ${ }^{34}$ In particular, we will study the cases $T(Q, K), T\left(Q_{\perp}, K_{\perp}\right)$, and $T\left(Q_{\|}, K_{\|}\right)$. The energy fluxes discussed in the previous section can be reobtained in terms of the shell-to-shell transfer function as

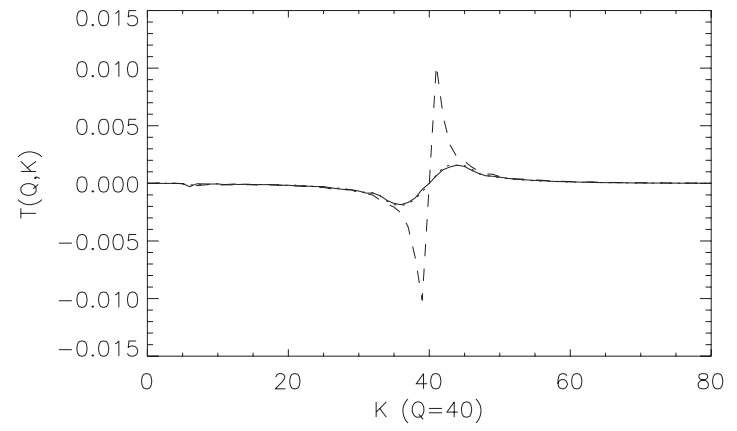

FIG. 8. Shell-to-shell transfer function $T(Q, K)$ at $Q=40$ for runs B1 (solid), B2 (dotted), and B3 (dashed) at late times.

$$
\Pi(k)=-\sum_{K=0}^{k} \sum_{Q} T(Q, K),
$$

where again the wavenumbers $k, K$, and $Q$ can correspond to different foliations of Fourier space depending on the subindex.

Note that for the definition of the shells a linear binning is used. Alternatively, the shells can be defined by a logarithmic binning of spectral space with intervals $\left(\gamma^{n} K_{0}, \gamma^{n+1} K_{0}\right]$ for some positive $\gamma>1$ and for integer $n$. However, logarithmic binning cannot distinguish transfer between linearly spaced neighbor shells (from the shell $K$ to the shell $K+1$ ) from the transfer between logarithmic neighbor shells (from $K$ to $\gamma K$ ). If the cascade is the result of interactions with the large-scale flow (e.g., with modes with wavenumber $k_{F}$ associated to the external forcing), the energy in a shell $K$ will be transferred to the shell $K+k_{F}$. Logarithmic binning does not distinguish this transfer from the transfer due to local triadic interactions that transfer the energy from $K$ to $\gamma K$. For this reason we use linear binning, but we note that care needs to be taken when using the word "scale" that implies in general a logarithmic division of the spectral space. The transfer among logarithmic shells can be reconstructed at any time later by summing over the linearly spaced shells.

Figure 8 shows the shell-to-shell transfer $T(Q, K)$ at $K$ $=40$ for runs B1, B2, and B3 at late times. The negative peak to the left indicates energy is transferred from these $K$-shells to the shell $Q=40$, while the positive peak to the right indicates energy goes from the $Q=40$ shell to those $K$-shells. In runs $\mathrm{B} 1$ and $\mathrm{B} 2$ the shell-to-shell transfer peaks at $|Q-K|$ $\approx k_{F}$. This was observed before in simulations of isotropic and homogeneous turbulence, ${ }^{32,33,35,36}$ and indicates that the energy transfer is local (the energy goes from a shell $Q$ to a nearby shell $K$, although the step in the energy cascade is independent of that scale and related to the forcing scale). In the context of the present study, it is worth mentioning that this is not a peculiarity of the TG forcing, and this transfer in nonrotating turbulence has been observed for other forcing functions, including isotropic delta correlated in time forcing. ${ }^{33,36}$ It is the result of individually strong triadic interaction involving the energy containing scale. As more triads are summed in order to obtain the shell-to-shell transfer and the energy flux, the functions become more local; at the 


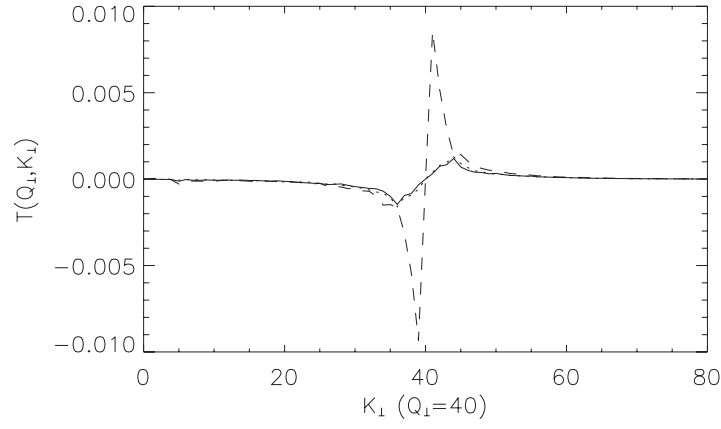

FIG. 9. Shell-to-shell transfer function $T\left(Q_{\perp}, K_{\perp}\right)$ at $Q_{\perp}=40$ for runs B1, B2, and B3. Labels are as in Fig. 8.

Reynolds number studied here, it can be shown that $\approx 60 \%$ of the energy flux in the nonrotating case is due to local interactions. $^{37}$

The shell-to-shell transfer in run B3 is markedly different and strongly peaks at $|Q-K| \approx 1$. The same effect is observed in $T\left(Q_{\perp}, K_{\perp}\right)$ shown in Fig. 9. This indicates that at late times in run B3, the direct transfer of energy at small scales is mediated by interactions with the largest scale in the system, the energy containing eddies with $k_{\perp} \approx 1$ (see Fig. $5)$. As a result, the timescale associated with the direct cascade of energy in $k_{\perp}$ increases (and its flux reduces, see Fig. 6) since the energy is transferred in smaller steps in Fourier space than in the case of the B1 and $\mathrm{B} 2$ runs.

The shell-to-shell transfer $T\left(Q_{\|}, K_{\|}\right)$at $Q_{\|}=40$ for the same runs is shown in Fig. 10. The dependence with the Rossby number of this transfer function is less drastic. In all runs, the transfer function $T\left(Q_{\|}, K_{\|}\right)$peaks at $\left|Q_{\|}-K_{\|}\right| \approx k_{F}$. Although, there is considerable amount of energy in the shell $k_{\|}=0$, the modes in this shell (that correspond to a pure 2D flow) do not cascade the energy in the $k_{\|}$axis since they are unable to stretch eddies in the z-direction. As a result, the cascade in this direction is only due to modes with $k_{\|}>0$ and the modes with $k_{\|}=k_{F}$ dominate. Note also that there is a drop in the amplitude of the transfer in run B3 for all shells except the ones satisfying $\left|Q_{\|}-K_{\|}\right|=k_{F}$. As a result, for small Rossby number the transfer of energy between shells with $Q_{\|}$ and $K_{\|}$is quenched except for the direct interactions with the external forcing. Most of the interactions responsible for the transfer of energy to small scales between different $k_{\|}$shells

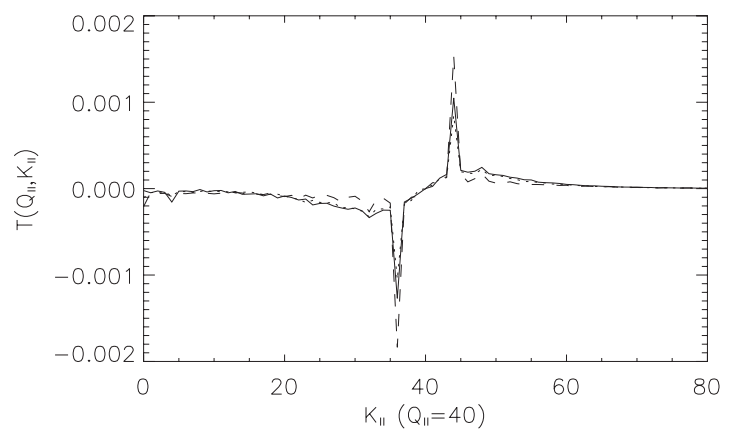

FIG. 10. Shell-to-shell transfer function $T\left(Q_{\|}, K_{\|}\right)$at $Q_{\|}=40$ for runs B1, B2, and B3. Labels are as in Fig. 8. Notice these transfers are roughly five times weaker than in the $\perp$ case. at small Ro are then interactions with the forcing. This is consistent with previous results showing that rotation reduces the turbulent energy transfer along the parallel direction.

Figure 11 shows the transfer functions $T\left(Q_{\perp}, K_{\perp}\right)$ and $T\left(Q_{\|}, K_{\|}\right)$in runs B1 and B3 for all values of $K$ and $Q$ up to 40. In all cases, the white (horizontal) and black (vertical) bands near $Q \approx k_{F}$ and $K \approx k_{F}$ indicate a small amount of energy injected by the external forcing that is directly transferred to all wavenumbers up to $\approx 30$. For $K$ and $Q$ larger than $k_{F}$, the figures confirm the results of the direct cascade of energy presented in Figs. 9 and 10: the bright diagonal bands below the $Q=K$ lines indicate energy is given to those $K$ shells, and the dark diagonal bands above $Q=K$ indicate energy is taken from those $K$ shells. As a result, when observed at constant $Q$ in the quadrant $K>k_{F}$ and $Q>k_{F}$, the figures again show the transfer of energy toward larger wavenumbers at scales smaller than the forcing.

For wavevectors perpendicular to $\boldsymbol{\Omega}$, as the Rossby number is decreased, the peaks in $T\left(Q_{\perp}, K_{\perp}\right)$ (the light and dark diagonal bands) move closer to the diagonal $K_{\perp}=Q_{\perp}$ [compare Figs. 11(a) and 11(b)], indicating the direct cascade in the perpendicular direction takes place in smaller $k$-steps given by the largest scale of the system. Note also how the diagonal bands become narrower in Fig. 11(b), indicating that, while the shell-to-shell transfer is still local, the triadic interactions are becoming more nonlocal as $\Omega$ increases.

For all wavenumbers, the energy in the parallel direction [see $T\left(Q_{\|}, K_{\|}\right)$in Figs. 11(c) and $11(\mathrm{~d})$ ] is transferred to smaller scales, and the cascade step does not depend on the Rossby number (as indicated by the distance between the maxima of the light and dark diagonal bands). However, all transfer except the transfer with $\left|Q_{\|}-K_{\|}\right|=k_{F}$ is strongly quenched in run B3. In other words, the diagonal bands become more localized in Fig. 11(d) [note how the gray bands surrounding the strong diagonal bands in Fig. 11(c) disappear], indicating all transfer in the parallel direction in run B3 is quenched except for the direct interactions with the external forcing.

The development of a nonlocal (and anisotropic) inverse transfer can be observed in Fig. 11(b) for $K_{\perp}<k_{f}$ (and for all values of $Q_{\perp}$ ) or in $Q_{\perp}<k_{f}$ (for all values of $K_{\perp}$ ). The transfer is inverse since below the diagonal $Q_{\perp}=K_{\perp}$ regions with negative (dark gray and black) $T\left(Q_{\perp}, K_{\perp}\right)$ can be observed, while above the diagonal light gray and white regions are found (note the change in sign above and below the diagonal when compared with the quadrant with $K$ and $Q$ larger than $k_{F}$, where the cascade is direct). As an illustration of this inverse cascade, energy is taken from, e.g., $K_{\perp}=20$ and transferred to shells with $Q_{\perp}<k_{F}$ (note the dark gray horizontal band for all values of $K_{\perp}$ and $Q_{\perp}<k_{F}$, and the light gray vertical band for all values of $Q_{\perp}$ and $K_{\perp}<k_{F}$ ). This transfer is nonlocal since the inverse transference takes place between disparate scales.

The nonlocal transfer of energy in rotating turbulence shares similarities with the inverse cascade of magnetic helicity in magnetohydrodynamics (MHD). ${ }^{38,39}$ Near the diagonal $Q_{\perp}=K_{\perp}$ and for $K_{\perp}<k_{f}$ and $Q_{\perp}<k_{f}$ the transfer is more complex (note how light and dark regions alternate 

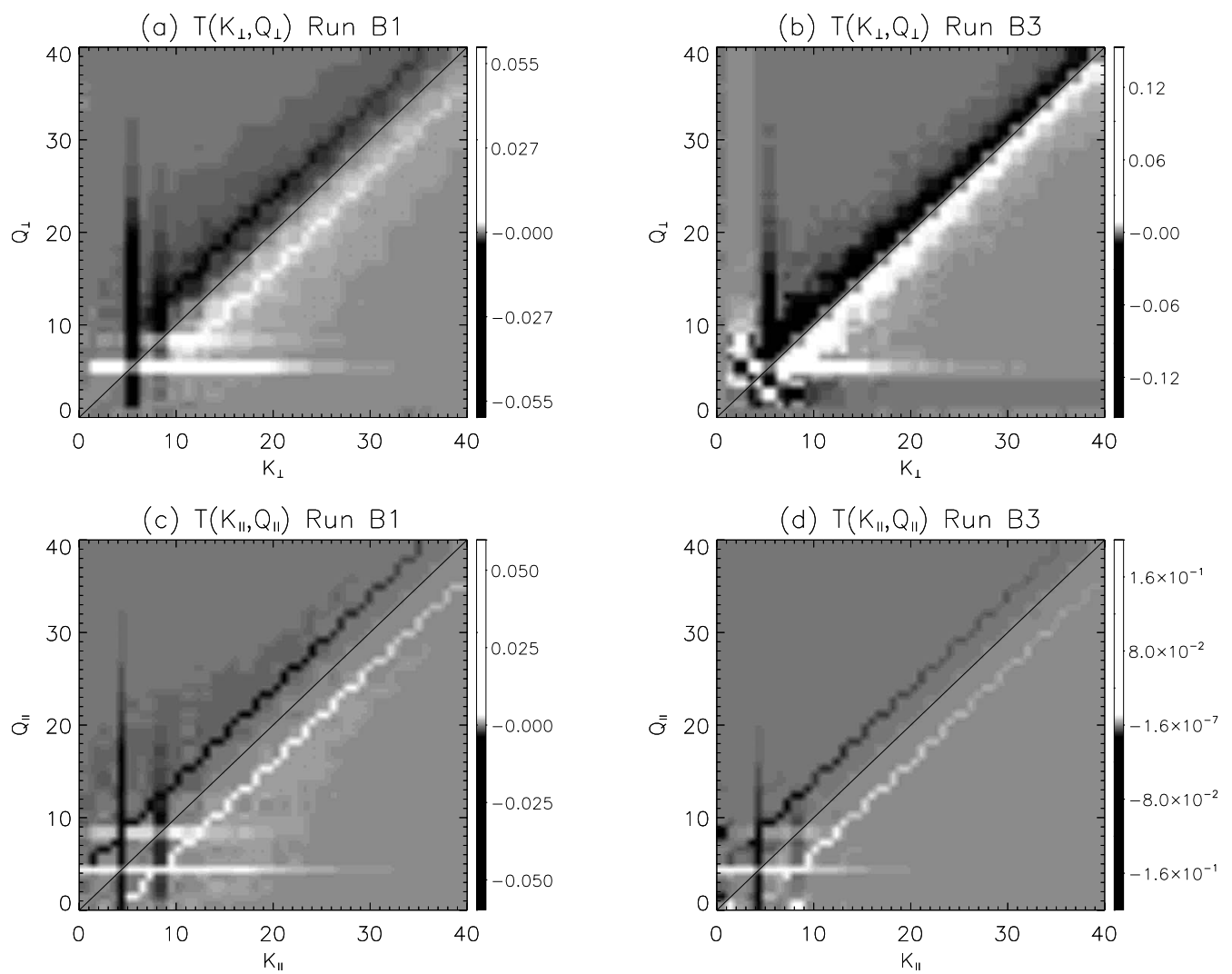

FIG. 11. Shell-to-shell energy transfer functions $T\left(Q_{\perp}, K_{\perp}\right)[(\mathrm{a})$ and (b) $]$ and $T\left(Q_{\|}, K_{\|}\right)[(\mathrm{c})$ and (d)] at late times in runs B1 [(a) and (c)] and B3 [(b) and (d)]. Notice the quenching of the transfer in case (d), except for the interactions with the forcing scale.

above and below the $Q_{\perp}=K_{\perp}$ diagonal). The inverse transfer superposes with a (smaller in net amplitude) direct local transfer (dark spots below and near the diagonal, and light spots above and near it, for $K_{\perp}$ and $Q_{\perp}$ smaller than $k_{F}$ ). This small direct transfer of energy at large scales is the result of a reflection of energy at $K=1$, and was also observed in studies of the inverse cascade of magnetic helicity in MHD. ${ }^{38}$ The reflection of energy in Fourier space when it reaches the largest scale in the box suggests that the late time evolution can be dependent on the boundary conditions, a property that was already observed in simulations of $2 \mathrm{D}$ turbulence. ${ }^{40-44}$ In our case, the simulations do not contain a large-scale dissipation mechanism (such as a hypoviscosity), and therefore energy piles up at the largest available scale until its growth is stopped by the (small-scale) dissipation.

\section{SCALING LAWS AND INTERMITTENCY}

In this section, we consider the anisotropic inertial range scaling of the runs in Table I as described by the longitudinal velocity increments in the direction perpendicular to rotation,

$$
\delta u\left(\mathbf{x}, \ell_{\perp}\right)=\hat{\mathbf{r}} \cdot[\mathbf{u}(\mathbf{x}+\ell \hat{\mathbf{r}})-\mathbf{u}(\mathbf{x})],
$$

where $\hat{\mathbf{r}}$ is a unit vector perpendicular to $\boldsymbol{\Omega}$. The longitudinal structure functions $S_{p}\left(\ell_{\perp}\right)$ (with displacements along $\ell_{\perp}$ ) can then be defined as

$$
S_{p}\left(\ell_{\perp}\right)=\left\langle\delta u\left(\mathbf{x}, \ell_{\perp}\right)^{p}\right\rangle,
$$

where the brackets denote spatial averaging, and where the dependence on $\mathbf{x}$ goes away because of homogeneity. If the flow is self-similar, we expect $S_{p}\left(\ell_{\perp}\right) \sim \ell_{\perp}^{\zeta_{p}}$, where $\zeta_{p}$ are the scaling exponents. In isotropic and homogeneous hydrodynamic turbulence, the Kármán-Howarth theorem implies $S_{3}(\ell) \sim \ell$, and the Kolmogorov energy spectrum follows from the assumption $S_{p}(\ell) \sim \ell^{p / 3}$. ${ }^{45}$ In practice, the spontaneous development of strong gradients in the small scales of a turbulent flow gives rise to corrections to this scaling, a phenomenon referred to as intermittency.

From dimensional analysis, if the energy spectrum at small scales in rotating turbulence is $E \sim k_{\perp}^{-2}$, we expect $S_{2}$ $\sim \ell_{\perp}$. Figure 12 shows the second order structure function for runs B1, B2, and B3 at late times outside the wave re-

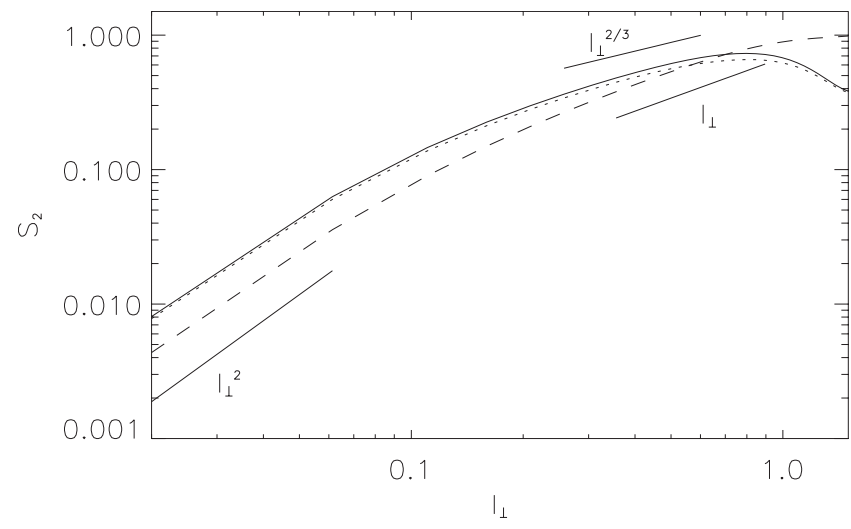

FIG. 12. Second order longitudinal structure function $S_{2}\left(\ell_{\perp}\right)$ (where $\ell_{\perp}$ denotes increments were taken in the direction perpendicular to $\boldsymbol{\Omega}$ ) for runs B1 (solid), B2 (dotted), and B3 (dashed). 


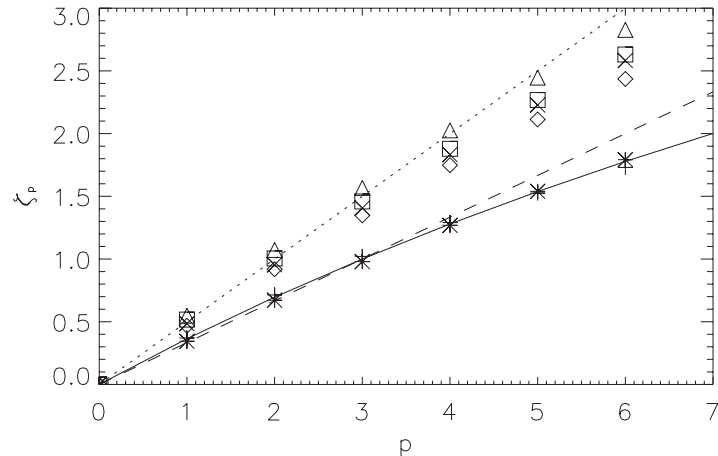

FIG. 13. Scaling exponents $\zeta_{p}$ for the steady state of runs B1 (+), B2 (*), and for run B3 at different times: $t \approx 20(\diamond), t \approx 25(\times), t \approx 30(\square)$, and $t$ $\approx 40(\triangle)$. The solid line corresponds to the scaling exponents given by the She-Lévêque model (Ref. 49), the dashed line is the Kolmogorov prediction $\zeta_{p}=p / 3$, and the dotted line is $\zeta_{p}=p / 2$. Note that in run $B_{3}$, as time evolves, the exponents approach the $p / 2$ scaling (see text).

gime when the turbulence has developed. At small scales for all runs, $S_{2} \sim \ell_{\perp}^{2}$, consistent with a smooth field in the dissipative range. At large scales, $S_{2}$ is larger for run B3 than for runs $\mathrm{B} 1$ and $\mathrm{B} 2$, a signature of the inverse cascade of energy and of the development of large-scale structures in the flow. The scaling of runs B1 and B2 at intermediate scales is compatible with the Kolmogorov spectrum, while the scaling in run B3 is consistent with the $\sim k_{\perp}^{-2}$ energy spectrum. Note that such a scaling can be understood as a slow down in the energy transfer rate because of interactions between waves and eddies as often assumed in phenomenological theories (see, e.g., Refs. 15, 17, and 46); such a slow down is consistent with the results of the transfer function presented in Sec. IV. Considering that the energy flux in the inertial range $\epsilon$ is slowed down by waves (see, e.g., Refs. 47 and 48 for the MHD case)

$$
\epsilon \sim \delta u_{\ell_{\perp}}^{2} \tau_{\Omega} / \tau_{\ell_{\perp}}^{2}
$$

where $\tau_{\Omega} \sim 1 / \Omega$, and $\tau_{\ell_{\perp}} \sim \ell_{\perp} / \delta u_{\ell_{\perp}}$ is the turn-over time of eddies in the plane perpendicular to $\boldsymbol{\Omega}$; the scaling

$$
\delta u_{\ell_{\perp}}^{2} \sim \ell_{\perp}
$$

follows.

Figure 13 shows the scaling exponents $\zeta_{p}$ up to order 6 computed in runs B1, B2, and B3. The scaling exponents are defined as the exponents in

$$
S_{p}\left(\ell_{\perp}\right) \sim \ell_{\perp}^{\zeta_{p}}
$$

in the inertial range associated to the direct cascade of energy (i.e., for $\left.\ell_{\perp}<L_{F}\right)$. Runs B1 and B2 behave as nonrotating turbulence, with Kolmogorov scaling $\left(\zeta_{2} \approx 2 / 3\right)$ and intermittency corrections (the prediction $\zeta_{p}=p / 3$ of Kolmogorov, and the model of intermittency in homogeneous and isotropic turbulence of She and Lévêque ${ }^{49}$ are shown in Fig. 13 as a reference). However, run B3 has a distinct behavior, with $\zeta_{2} \approx 1$. As time evolves in this run, and the energy piles up at $k_{\perp} \approx 1$, the second order scaling exponent slowly converges to this value. Low order moments follow the curve $\zeta_{p}=p / 2$, but high order moments deviate from the straight line.

Similar results were found in an experimental study of a turbulent flow between two corotating disks ${ }^{28}$ (see Fig. 4.18 in Ref. 25). The resulting experimental flow shares similarities with the TG flow studied here. In the experiment, a transition from isotropic and homogeneous turbulence scaling to a $\zeta_{p}=p / 2$ scaling was observed as measurements were made closer to the intense large-scale vortex formed in the gap between the two disks.

The level of intermittency in the flow in all these runs can be measured in terms of $\mu=2 \zeta_{3}-\zeta_{6}$. This quantity, together with the integral scales of the flow (based on the parallel and perpendicular energy spectra), the Taylor scale, and the micro-Rossby number (based on the Taylor scale of the flow),

$$
\operatorname{Ro}_{\lambda}=\frac{U}{2 \Omega \lambda},
$$

are given in Table II for the runs in set B at different times. The value of the micro-Rossby number plays a central role in the determination of the velocity derivative skewness and the inhibition of the energy cascade in rotating turbulence, as discussed in Ref. 6. In addition, its value is also important in the development of anisotropies through nonlinear interactions, as is discussed in more detail in Sec. VI.

It can be seen that at late times run B3 evolves toward an anisotropic state in the large scales, with $L_{\perp} / L_{\|} \approx 1.5$ (see Sec. VI for more details). However, at small scales the flow seems more isotropic and at late times $(t \approx 40)$ in this run $\lambda_{\perp} / \lambda_{\|} \approx 0.8$. The micro-Rossby number in runs B1, B2, and B3 takes different values, in the range of 0.11-3.7. However, the value of $\mu$ is, within error bars, approximately the same for all the runs. As a result, the intermittency in the direct

TABLE II. Characteristic scales and dimensionless numbers of the runs in set B. $t$ is the time, $L_{\|}$and $L_{\perp}$ are the integral scales using respectively the $E\left(k_{\|}\right)$and $E\left(k_{\perp}\right)$ spectra, $\lambda$ is the isotropic Taylor scale, $\operatorname{Ro}_{\lambda}$ is the micro-Rossby number based on the Taylor scale, and $\mu=2 \zeta_{3}-\zeta_{6}$.

\begin{tabular}{lllllll}
\hline \hline Run & $t$ & $L_{\|}$ & $L_{\perp}$ & $\lambda$ & $\operatorname{Ro}_{\lambda}$ & $\mu$ \\
\hline B1 & 16 & 1.5 & 0.9 & 0.29 & 3.70 & $0.23 \pm 0.01$ \\
B2 & 24 & 1.6 & 0.9 & 0.31 & 0.91 & $0.24 \pm 0.01$ \\
B3 & 20 & 2.6 & 1.2 & 0.50 & 0.12 & $0.19 \pm 0.02$ \\
B3 & 25 & 2.4 & 1.5 & 0.55 & 0.11 & $0.26 \pm 0.02$ \\
B3 & 30 & 2.1 & 1.7 & 0.59 & 0.12 & $0.26 \pm 0.05$ \\
B3 & 40 & 1.9 & 2.8 & 0.53 & 0.33 & $0.24 \pm 0.02$ \\
\hline \hline
\end{tabular}




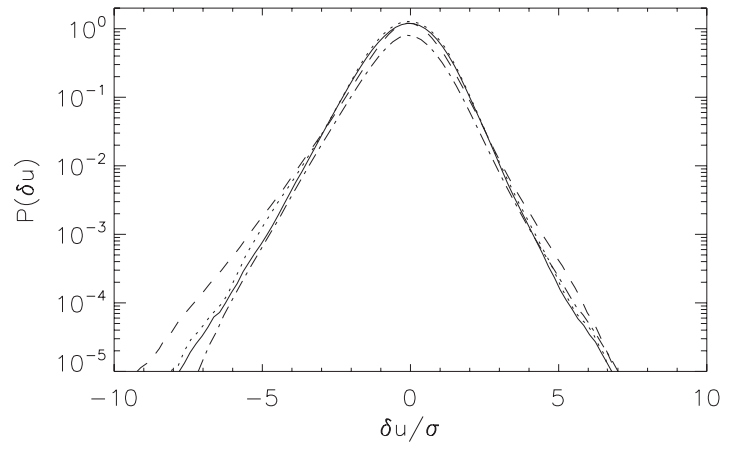

FIG. 14. pdf of the longitudinal velocity increments $\left(\ell_{\perp}=3 \eta\right)$ for run B3 at different times: $t \approx 20$ (solid), $t \approx 25$ (dotted), $t \approx 30$ (dashed), and $t \approx 40$ (dashed-dotted); $\eta$ is the Kolmogorov dissipative length and $\sigma$ is the root mean square deviation of the velocity increments.

cascade of energy in a rotating flow seems to be independent of the Rossby number Ro and of the micro-Rossby number $\mathrm{Ro}_{\lambda}$ (in the range of Rossby numbers studied).

Finally, Fig. 14 shows the time evolution of the probability density function (pdf) of the longitudinal velocity increments in run B3. Increments in the direction perpendicular to $\boldsymbol{\Omega}$ were computed, and the increment was taken equal to three times the Kolmogorov dissipation scale $\eta$ in each run. The velocity increments in each run were normalized by their corresponding root mean square deviation $\sigma$. In agreement with the level of intermittency observed in the scaling exponents, the pdfs show exponential tails indicating a larger than Gaussian probability of large gradients to occur in the small scales. The amplitude of the tails of the pdfs as a function of $\delta u / \sigma$ does not change significantly with time. Moreover, the root mean square deviation $\sigma$ of the velocity increments $\delta u$ increases with time. So if the pdfs are plotted versus $\delta u$ (instead of versus $\delta u / \sigma$ ), the pdfs actually become wider at later times. This effect can be understood considering that once the inverse cascade of energy sets in, the total energy in the flow as a function of time increases.

\section{STRUCTURES AND ANISOTROPY}

The intermittency reported in Sec. $\mathrm{V}$ in the scaling exponents and the pdfs of velocity increments indicates that even after the inverse cascade sets in, the flow develops strong velocity gradients in the small scales. In this section, we present visualizations of the flow and consider the structures that emerge.

Figure 15 shows a three-dimensional rendering of the vorticity intensity in half of the computational domain (256 $\times 512 \times 512$ grid points) at late times. The top view corresponds to the subvolume in the direction of the axis of rotation. Only regions with strong vorticity are shown. Note that the flow is anisotropic and quasi-2D, as it is clear from the top view. In the bottom view, the development in the flow of large-scale columnlike structures can be seen. However, the columns display small-scale structures with thin vortex filaments. These filaments seem to be ordered according to the large-scale pattern. The presence of regions with strong vorticity even when the Rossby number is small enough for the
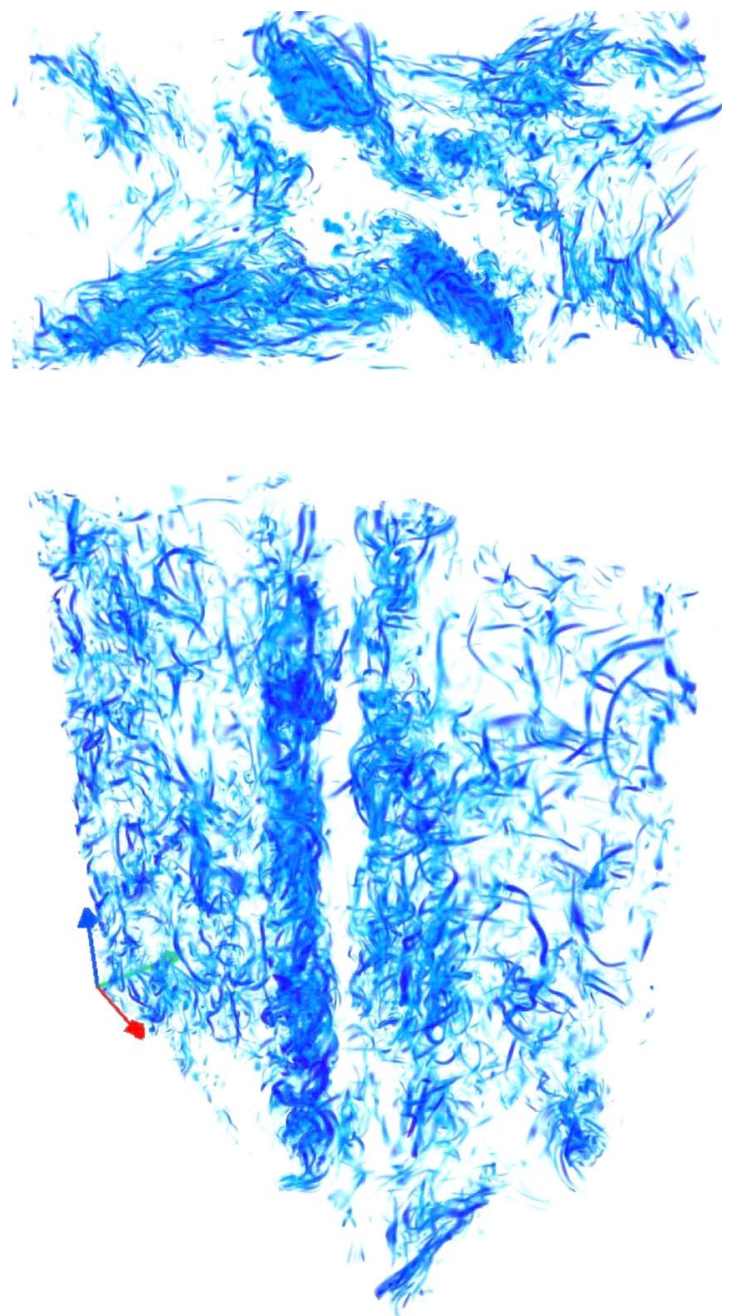

FIG. 15. (Color online) Three-dimensional rendering of the vorticity intensity in a subvolume of $256 \times 512 \times 512$ grid points for run B2. The top view shows the subvolume in the direction of the axis of rotation; in the bottom view, the light gray (red) and dark gray (blue) arrows indicate, respectively, the $x$ and $z$ axis. Note the large-scale columns made up of smaller-scale intense vortices typical of three-dimensional turbulence.

inverse cascade of energy to develop can be expected from the results shown in Figs. 13 and 14, linked to the intermittency of the flow.

The development of anisotropies in the runs with small Rossby number is apparent from Fig. 15. Table III gives a list of different measures of anisotropy commonly used in literature, for all runs at different times. According to Refs. 6 and 50 , anisotropies develop in rotating flows through nonlinear interactions when the Rossby number Ro $<1$ and when the micro-Rossby number $\operatorname{Ro}_{\omega}>1$. The micro-Rossby number $\mathrm{Ro}_{\omega}$ is the ratio of rms vorticity to background vorticity (rotation),

$$
\operatorname{Ro}_{\omega}=\frac{\omega}{2 \Omega},
$$

and scales as $\mathrm{Ro}_{\lambda}$ except for prefactors. If the macro-Rossby number Ro is too large, no inverse cascade is observed in the simulations. If the micro-Rossby number is too small, nonlinear interactions are completely damped and no transition develops. It is worth noting that the actual values for the 
TABLE III. Different measures of anisotropy in all runs at different times. $t$ is the time, $\mathrm{Ro}_{\omega}$ is the microRossby number based on the rms vorticity, $L_{\perp} / L_{\|}$is the ratio of perpendicular to parallel integral scales, $\tan ^{2} \theta$ is the square tangent of the Shebalin angle, and $E\left(k_{\|}=0\right) / E$ is the ratio of energy in all modes with $k_{\|}=0$ to the total energy.

\begin{tabular}{lccccc}
\hline \hline Run & $t$ & $\mathrm{Ro}_{\omega}$ & $L_{\perp} / L_{\|}$ & $\tan ^{2} \theta$ & $E\left(k_{\|}=0\right) / E$ \\
\hline $\mathrm{A} 1$ & 45 & 65.2 & 0.7 & 1.74 & 0.05 \\
$\mathrm{~A} 2$ & 45 & 12.7 & 0.7 & 1.78 & 0.07 \\
$\mathrm{~A} 3$ & 45 & 5.89 & 0.7 & 1.98 & 0.13 \\
$\mathrm{~A} 4$ & 45 & 3.18 & 0.6 & 2.55 & 0.19 \\
$\mathrm{~A} 5$ & 150 & 1.23 & 0.5 & 4.01 & 0.23 \\
$\mathrm{~A} 6$ & 110 & 0.22 & 0.4 & 12.3 & 0.15 \\
$\mathrm{~A} 6$ & 150 & 0.48 & 1.5 & 7.65 & 0.82 \\
$\mathrm{~A} 6$ & 185 & 1.12 & 1.9 & 3.80 & 0.88 \\
B1 & 16 & 22.4 & 0.6 & 1.77 & 0.03 \\
B2 & 24 & 5.61 & 0.6 & 2.04 & 0.11 \\
B3 & 20 & 0.77 & 0.5 & 6.22 & 0.36 \\
B3 & 25 & 0.67 & 0.6 & 6.58 & 0.55 \\
B3 & 30 & 0.76 & 0.8 & 5.87 & 0.62 \\
B3 & 40 & 1.35 & 1.5 & 3.82 & 0.66 \\
\hline \hline
\end{tabular}

transition to occur can depend on the particular flow studied.

The development of anisotropies in rotating turbulence has been quantified in Refs. 5, 6, and 20 using the parallel and perpendicular integral scales. The ratio of these two length scales is given in Table III. For Ro $\leqq 0.35, L_{\perp} / L_{\|}$ $\approx 0.7$ (runs A1-A3, B1, and B2); in the absence of rotation the flow under TG forcing develops a slightly larger integral scale in the $z$ direction. However, in runs A4-A6 and B3, the ratio $L_{\perp} / L_{\|}$increases and becomes larger than 1 as Ro is decreased. In runs A6 and B3, this ratio is observed first to decrease (just before the inverse cascade starts) to finally increase monotonically, reaching $L_{\perp} / L_{\|} \approx 1.9$ in run A6 at $t$ $\approx 185$.

The integral scale ratio measures anisotropy in the energy containing scale. To quantify small-scale anisotropy, the so-called Shebalin angles were introduced in MHD turbulence, ${ }^{51,52}$

$$
\tan ^{2}(\theta)=2 \lim _{\ell \rightarrow 0} \frac{S_{2}\left(\ell_{\perp}\right)}{S_{2}\left(\ell_{\|}\right)}=2 \frac{\sum_{k_{\perp}} k_{\perp}^{2} E\left(k_{\perp}\right)}{\sum_{k_{\|}} k_{\|}^{2} E\left(k_{\|}\right)},
$$

where $\ell_{\|}$denotes displacements along $\boldsymbol{\Omega}$. The angle $\theta$ measures the spectral anisotropy level, and the case $\tan ^{2}(\theta)=2$ corresponds to an isotropic flow. It gives only a global measure of small-scale anisotropy, and is a byproduct of axisymmetric energy spectra (see Refs. 6, 10, and 53). Values of $\tan ^{2}(\theta)$ for all runs are listed in Table III. Runs A1 and B1 have $\tan ^{2}(\theta) \approx 1.7$, a value close to isotropy and with a small anisotropy in the $z$ direction. As the Rossby number is decreased, this tendency is reverted and $\tan ^{2}(\theta)$ becomes larger than 2. For runs $\mathrm{A} 6$ and $\mathrm{B} 3$ the value of $\tan ^{2}(\theta)$ is given for different times. As time evolves in these runs, the anisotropy is maximum just before the inverse cascade starts, and then $\tan ^{2}(\theta)$ decreases slowly to saturate near $\approx 3.8$.

The spectral anisotropy has also been studied through the axisymmetric energy spectrum $e\left(k_{\|}, k_{\perp}\right)$ (as well as other second order quantities; see, e.g., Refs. 5, 6, 54, and 55). For a $2 \mathrm{D}$ flow,

$$
e\left(k_{\|}, k_{\perp}\right)=\frac{E\left(k_{\perp}\right)}{2 \pi k_{\perp}} \delta\left(k_{\|}\right)
$$

In rotating turbulence, a pure $2 \mathrm{D}$ state is never reached; ${ }^{10}$ however, a strong anisotropy develops with a steeper spectrum in the parallel direction ${ }^{7,10,53}$ consistent with an integrable singularity at $k_{\|}=0$. As an indication of this tendency, in Table III we give the ratio of energy in all modes with $k_{\|}=0$ to the total energy $E\left(k_{\|}=0\right) / E$; in the purely $2 \mathrm{D}$ case this ratio is equal to one. Runs $\mathrm{A} 1$ and $\mathrm{B} 1$ have small $E\left(k_{\|}\right.$ $=0) / E$, and the ratio increases as Ro is decreased, reaching $E\left(k_{\|}=0\right) / E \approx 0.88$ at late times in run $\mathrm{A} 6(\mathrm{Ro} \approx 0.03)$ and $E\left(k_{\|}=0\right) / E \approx 0.66$ in run $\mathrm{B} 3(\mathrm{Ro} \approx 0.07)$.

As the energy, the helicity is conserved in ideal rotating flows. However, the distribution of helicity seems to be more isotropic and homogeneous than the other quantities studied. As an example, the local relative helicity $\boldsymbol{\omega} \cdot \mathbf{u} /(|\boldsymbol{\omega} \| \mathbf{u}|)$ is shown in Fig. 16, for the same subvolume as in Fig. 15. Unlike in isotropic and homogeneous turbulence, regions of strong vorticity are not correlated with regions of strong relative helicity. This is because while in isotropic and homogeneous turbulence quenching of nonlinear interactions occurs through alignment of velocity and vorticity, in rotating turbulence it results from phase-mixing and it is not necessarily linked to regions of strong helicity. The net helicity over the entire box averages to zero, and local regions with positive and negative helicity fluctuations, although ubiquitously strong, show a more isotropic and homogeneous distribution than the vorticity and velocity intensities (see, e.g., Fig. 15). The study of the reasons for this behavior are left for a future work. 

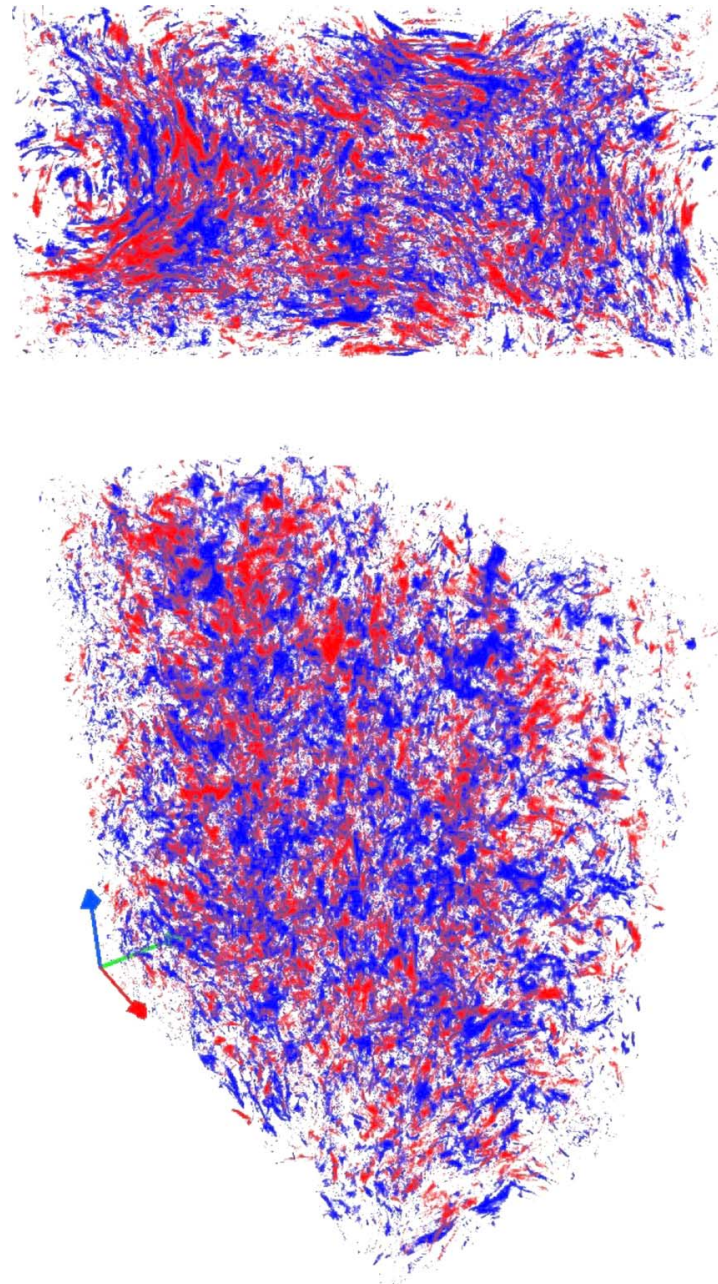

FIG. 16. (Color online) Rendering of relative helicity in the same subvolume as in Fig. 15 and with the same viewpoints. Dark gray (blue) corresponds to positive helicity, and light gray (red) to regions with negative helicity. Only regions with $|\boldsymbol{\omega} \cdot \mathbf{u}| /(|\boldsymbol{\omega} \| \mathbf{u}|)>0.95$ are shown, i.e., for strong alignment between the velocity and vorticity, which appears ubiquitous and not confined to strong vorticity regions as in the nonrotating case.

\section{CONCLUSIONS}

In this work, we presented results of the study of the turbulent scaling laws and energy transfer in direct numerical simulations of rotating flows in periodic domains. Spatial resolutions of $256^{3}$ (set A) and of $512^{3}$ grid points (set B) were used, while moderate Rossby numbers (down to Ro $\approx 0.03$ ) and large Reynolds numbers (up to $\operatorname{Re} \approx 1100$ ) were considered, with enough scale separation to observe both a direct and an inverse cascade of energy when the rotation was strong enough. Runs in set A were started from a fluid at rest, while runs in set B were restarted from a previous state of homogeneous turbulence. In the former case, for Ro $\approx 0.1$, a long transient was found in which the energy dissipation is small, as well as the energy flux to smaller scales. During this transient, the energy spectrum has a wide but steep spectrum, and its slope monotonously increases as a function of time. After turbulence sets in and the inverse cascade of energy develops, the energy spectrum evolves toward a $E \sim k_{\perp}^{-2}$ scaling at scales smaller than the forcing scale. This late time evolution is observed in both sets of runs.

At late times, the energy flux in runs A5, A6, and B3 corresponding to strong rotation indicates an inverse cascade of energy in $k_{\perp}$ at scales larger than the forcing scale, together with a direct cascade of energy at smaller scales. The net flux to small scales decreases as the Rossby number decreases, while the amplitude of the flux to large scales increases. No inverse cascade is observed in $k_{\|}$. This is consistent with the explanation of the observed tendency toward two dimensionalization through resonant interactions ${ }^{4,5}$ and not through an inverse cascade in $k_{\|}$. These cascades were confirmed by the study of the shell-to-shell energy transfer. The direct transfer of energy at scales smaller than the forcing is local, although in the runs with small Rossby number the transfer in $k_{\perp}$ is significantly slowed down. In this direction, the energy is transferred between shells $K_{\perp}$ and $Q_{\perp}$ with small steps given by $\left|Q_{\perp}-K_{\perp}\right| \approx 1$. As a result, the direct transfer of energy in $k_{\perp}$ at small scales is mediated by interactions with the largest scale in the system, the energy containing eddies with $k_{\perp} \approx 1$. The time scale associated to the direct cascade in $k_{\perp}$ then increases, and its flux reduces. In $k_{\|}$the transfer is direct at all scales, and a larger component than in the case of nonrotating turbulence is due to interactions with the forcing scale. These results are in good agreement with phenomenological derivations of the energy spectrum in rotating turbulence that assume a slow down in the energy transfer rate because of interactions between waves and eddies. ${ }^{15,17}$ The nonlocal interactions also lead to the development of anisotropies in the flow. ${ }^{4,5}$

The inverse cascade of energy that develops at scales larger than the forcing scale, in runs A5, A6, and B3 is nonlocal, in the sense that the transfer of energy associated to this cascade takes place between disparate shells in Fourier space. At late times, the inverse transfer superposes at the same scales with a (smaller in amplitude) direct local transfer of energy. This small direct transfer of energy at large scales is the result of a reflection at $k_{\perp}=1$, when the peak of energy reaches the largest scale in the box. Consequently, the late time evolution of simulations of rotating turbulence may depend on the boundary conditions used, a property already observed in simulations of nonrotating $2 \mathrm{D}$ turbulence, ${ }^{40-44}$ and on the use or not of a friction or dissipative term at large scales.

The study of structure functions in the direct cascade range shows that the second order scaling exponent for increments perpendicular to the rotation in runs with small Ro is $\zeta_{2} \approx 1$, in agreement with the evaluation of the energy spectrum spectral index. Low order moments follow the curve $\zeta_{p}=p / 2$ but high order moments deviate from this law, an indication of intermittency. The level of intermittency in the direct cascade of energy, as measured by the exponent $\mu=2 \zeta_{3}-\zeta_{6}$, is the same for runs with and without rotation. The spontaneous formation of strong gradients in the small scales is further confirmed by pdfs of the velocity increments and by visualization of regions of strong vorticity in the flow.

More separation of scales is needed to study the intermittency in the inverse cascade of energy. Because of its relation to small-scale gradients, intermittency is believed to 
be associated only with the forward cascade of energy. The intermittency phenomenon is not observed in the velocity field in 2D turbulence for which the conservation of vorticity leads to an inverse energy cascade to the large scales, ${ }^{56,57}$ although intermittency in the vorticity (which cascades directly to small scales) is observed. It is unclear how the dual cascade of energy (toward both small and large scales) in rotating turbulence affects the intermittency in the inverse cascade range. While intermittency is associated with smallscale events, in many cases the strong events can affect the dynamics of the large scales, specially in systems close to criticality; as an example, intermittency is a possible explanation for the occurrence of extended minima in solar activity ${ }^{58,59}$ it is also known to affect the transport of momentum in atmospheric surface layers. ${ }^{60}$

\section{ACKNOWLEDGMENTS}

The authors would like to express their gratitude to J. R. Herring and J. J. Tribbia for their careful reading of the manuscript. Computer time was provided by NCAR. P.D.M. is a member of the Carrera del Investigador Científico of CONICET. A.A. acknowledges support from the Observatoire de la Côte d'Azur and Rotary Club's district 1730. The NSF Grant No. CMG-0327888 at NCAR supported this work in part. NCAR is sponsored by the NSF. Threedimensional visualizations of the flows were done using VAPOR, a software for interactive visualization and analysis of terascale data sets. ${ }^{61}$

${ }^{1}$ J. Pedlosky, Geophysical Fluid Dynamics (Springer, Berlin, 1986).

${ }^{2}$ M. S. Miesch, J. R. Elliott, J. T. T. L. Clune, G. A. Glatzmaier, and P. A. Gilman, "Three-dimensional spherical simulations of solar convection. I. Differential rotation and pattern evolution achieved with laminar and turbulent states," Astrophys. J. 532, 593 (2000).

${ }^{3}$ H. P. Greenspan, The Theory of Rotating Fluids (Cambridge University Press, Cambridge, 1968).

${ }^{4}$ F. Waleffe, "Inertial transfers in the helical decomposition," Phys. Fluids A 5, 677 (1993).

${ }^{5}$ C. Cambon and L. Jacquin, "Spectral approach to non-isotropic turbulence subjected to rotation," J. Fluid Mech. 202, 295 (1989).

${ }^{6}$ C. Cambon, N. N. Mansour, and F. S. Godeferd, "Energy transfer in rotating turbulence," J. Fluid Mech. 337, 303 (1997).

${ }^{7}$ S. Galtier, "Weak inertial-wave turbulence theory," Phys. Rev. E 68 , 015301 (2003).

${ }^{8}$ P. F. Embid and A. Majda, "Averaging over fast gravity waves for geophysics flows with arbitrary potential vorticity," Commun. Partial Differ. Equ. 21, 619 (1996).

${ }^{9}$ A. Babin, A. Mahalov, and B. Nicolaenko, "Global splitting, integrability and regularity of three-dimensional Euler and Navier-Stokes equations for uniformly rotating fluids," Eur. J. Mech. B/Fluids 15, 291 (1996).

${ }^{10}$ F. Bellet, F. S. Godeferd, J. F. Scott, and C. Cambon, "Wave turbulence in rapidly rotating flows," J. Fluid Mech. 562, 83 (2006).

${ }^{11}$ L. M. Smith and Y. Lee, "On near resonances and symmetry breaking in forced rotating flows at moderate rossby number," J. Fluid Mech. 535, 111 (2005)

${ }^{12}$ P. J. Staplehurst, P. A. Davidson, and S. B. Dalziel, "Structure formation in homogeneous freely decaying rotating turbulence," J. Fluid Mech. 598, 81 (2008).

${ }^{13}$ L. J. A. van Bokhoven, C. Cambon, L. Liechtenstein, F. S. Godeferd, and H. J. H. Clercx, "Refined vorticity statistics of decaying rotating threedimensional turbulence," J. Turbul. 9, N6 (2008).

${ }^{14} \mathrm{O}$. Zeman, "A note on the spectra and decay of rotating homogeneous turbulence," Phys. Fluids 6, 3221 (1994).

${ }^{15}$ Y. Zhou, "A phenomenological treatment of rotating turbulence," Phys. Fluids 7, 2092 (1995).
${ }^{16}$ V. M. Canuto and M. S. Dubovikov, "A dynamical model for turbulence. V. The effect of rotation," Phys. Fluids 9, 2132 (1997).

${ }^{17}$ W.-C. Müller and M. Thiele, "Scaling and energy transfer in rotating turbulence," Europhys. Lett. 77, 34003 (2007).

${ }^{18}$ J. Bardina, J. H. Ferziger, and R. S. Rogallo, "Effect of rotation on isotropic turbulence: computation and modeling," J. Fluid Mech. 154, 321 (1985).

${ }^{19}$ M. Hossain, "Reduction in the dimensionality of turbulence due to a strong rotation," Phys. Fluids 6, 1077 (1994).

${ }^{20} \mathrm{P}$. Bartello, O. Métais, and M. Lesieur, "Coherent structures in rotating three-dimensional turbulence," J. Fluid Mech. 273, 1 (1994).

${ }^{21}$ L. M. Smith, J. R. Chasnov, and F. Waleffe, "Crossover from two-to three-dimensional turbulence," Phys. Rev. Lett. 77, 2467 (1996).

${ }^{22}$ L. M. Smith and F. Waleffe, "Transfer of energy to two-dimensional large scales in forced, rotating three-dimensional turbulence," Phys. Fluids 11, 1608 (1999).

${ }^{23}$ P. K. Yeung and Y. Zhou, "Numerical study of rotating turbulence with external forcing," Phys. Fluids 10, 2895 (1998).

${ }^{24}$ Q. Chen, S. Chen, G. L. Eyink, and D. Holm, "Resonant interactions in rotating homogeneous three-dimensional turbulence," J. Fluid Mech. 542, 139 (2005).

${ }^{25}$ P. Sagaut and C. Cambon, Homogeneous Turbulence Dynamics (Cambridge University Press, Cambridge, 2008).

${ }^{26}$ G. I. Taylor and A. E. Green, "Mechanism of the production of small eddies from large ones," Proc. R. Soc. London, Ser. A 158, 499 (1937).

${ }^{27}$ R. H. Morf, S. A. Orszag, and U. Frisch, "Spontaneous singularity in three-dimensional, inviscid, incompressible flow," Phys. Rev. Lett. 44, 572 (1980).

${ }^{28}$ C. Simand, F. Chillà, and J.-F. Pinton, "Study of inhomogeneous turbulence in the closed flow between corotating disks," Europhys. Lett. 49, 336 (2000).

${ }^{29}$ R. Monchaux, M. Berhanu, M. Bourgoin, M. Moulin, Ph. Odier, J.-F. Pinton, R. Volk, S. Fauve, N. Mordant, F. Pétrélis, A. Chiffaudel, F. Daviaud, B. Dubrulle, C. Gasquet, L. Marié, and F. Ravelet, "Generation of a magnetic field by dynamo action in a turbulent flow of liquid sodium," Phys. Rev. Lett. 98, 044502 (2007).

${ }^{30}$ A. Alexakis, P. D. Mininni, and A. Pouquet, "Shell-to-shell energy transfer in magnetohydrodynamics. I. steady state turbulence," Phys. Rev. E 72, 046301 (2005).

${ }^{31}$ P. D. Mininni, A. Alexakis, and A. Pouquet, "Shell-to-shell energy transfer in magnetohydrodynamics. II. kinematic dynamo," Phys. Rev. E 72, 046302 (2005).

${ }^{32}$ A. Alexakis, P. D. Mininni, and A. Pouquet, "Imprint of large-scale flows on turbulence," Phys. Rev. Lett. 95, 264503 (2005).

${ }^{33}$ P. D. Mininni, A. Alexakis, and A. Pouquet, "Large-scale flow effects, energy transfer, and self-similarity on turbulence," Phys. Rev. E 74, 016303 (2006).

${ }^{34}$ A. Alexakis, B. Bigot, H. Politano, and S. Galtier, "Anisotropic fluxes and nonlocal interactions in magnetohydrodynamic turbulence," Phys. Rev. E 76, 056313 (2007).

${ }^{35}$ J. A. Domaradzki, "Analysis of energy transfer in direct numerical simulations of isotropic turbulence," Phys. Fluids 31, 2747 (1988).

${ }^{36}$ J. A. Domaradzki and R. S. Rogallo, "Local energy transfer and nonlocal interactions in homogeneous, isotropic turbulence," Phys. Fluids A 2, 413 (1990).

${ }^{37}$ P. D. Mininni, A. Alexakis, and A. Pouquet, "Nonlocal interactions in hydrodynamic turbulence at high Reynolds numbers: The slow emergence of scaling laws," Phys. Rev. E 77, 036306 (2008).

${ }^{38}$ A. Alexakis, P. D. Mininni, and A. Pouquet, "On the inverse cascade of magnetic helicity," Astrophys. J. 640, 335 (2006).

${ }^{39}$ A. Alexakis, P. D. Mininni, and A. Pouquet, "Turbulent cascades, transfer, and scale interactions in magnetohydrodynamics," New J. Phys. 9, 298 (2007).

${ }^{40}$ J. C. McWilliams, "The emergence of isolated coherent vortices in turbulent flow," J. Fluid Mech. 146, 21 (1984).

${ }^{41}$ M. E. Brachet, M. Meneguzzi, and P. L. Sulem, "Small-scale dynamics of high-Reynolds-number two-dimensional turbulence," Phys. Rev. Lett. 57, 683 (1986).

${ }^{42}$ A. Babiano, C. Basdevant, B. Legras, and R. Sadourny, "Vorticity and passive-scalar dynamics in two-dimensional turbulence," J. Fluid Mech. 183, 379 (1987).

${ }^{43}$ R. Benzi, S. Patarnello, and P. Santangelo, "On the statistical properties of two-dimensional decaying turbulence," Europhys. Lett. 3, 811 (1987). 
${ }^{44}$ M. E. Maltrud and G. K. Vallis, "Energy spectra and coherent structures in forced two-dimensional and beta-plane turbulence," J. Fluid Mech. 228, 321 (1991).

${ }^{45}$ U. Frisch, Turbulence: The Legacy of A.N. Kolmogorov (Cambridge University Press, Cambridge, 1995).

${ }^{46}$ P. D. Mininni and A. Pouquet, "Energy spectra stemming from interactions of Alfvén waves and turbulent eddies," Phys. Rev. Lett. 99, 254502 (2007).

${ }^{47}$ P. S. Iroshnikov, "Turbulence of a conducting fluid in a strong magnetic field," Sov. Astron. 7, 566 (1963).

${ }^{48}$ R. H. Kraichnan, "Inertial-range spectrum of hydromagnetic turbulence," Phys. Fluids 8, 1385 (1965).

${ }^{49}$ Z. S. She and E. Lévêque, "Universal scaling laws in fully developed turbulence," Phys. Rev. Lett. 72, 336 (1994).

${ }^{50}$ L. Jacquin, O. Leuchter, C. Cambon, and J. Mathieu, "Homogeneous turbulence in the presence of rotation," J. Fluid Mech. 220, 1 (1990).

${ }^{51}$ J. V. Shebalin, W. H. Matthaeus, and D. Montgomery, "Anisotropy in MHD turbulence due to a mean magnetic field," J. Plasma Phys. 29, 525 (1983).

${ }^{52}$ L. J. Milano, W. H. Matthaeus, P. Dmitruk, and D. C. Montgomery, "Local anisotropy in incompressible magnetohydrodynamic turbulence," Phys. Plasmas 8, 2673 (2001).
${ }^{53}$ L. Liechtenstein, F. S. Godeferd, and C. Cambon, "Nonlinear formation of structures in rotating stratified turbulence," J. Turbul. 6, 1 (2005).

${ }^{54}$ Y. Morinishi, K. Nakabayashi, and S. Ren, "Dynamics of anisotropy on decaying homogeneous turbulence subjected to system rotation," Phys. Fluids 13, 2912 (2001).

${ }^{55} \mathrm{X}$. Yang and J. A. Domaradzki, "LES of decaying rotating turbulence," Phys. Fluids 16, 4088 (2004).

${ }^{56}$ R. Benzi and R. Scardovelli, "Intermittency of two-dimensional decaying turbulence," Europhys. Lett. 29, 371 (1995).

${ }^{57} \mathrm{G}$. Boffetta, "Energy and enstrophy fluxes in the double cascade of twodimensional turbulence," J. Fluid Mech. 589, 253 (2007).

${ }^{58} \mathrm{P}$. Charbonneau, "Multiperiodicity, chaos, and intermittency in a reduced model of the solar cycle," Sol. Phys. 199, 385 (2001).

${ }^{59}$ P. D. Mininni, D. O. Gómez, and G. B. Mindlin, "Biorthogonal decomposition techniques unveil the nature of the irregularities observed in the solar cycle," Phys. Rev. Lett. 89, 061101 (2002).

${ }^{60}$ J. R. Kulkarni, L. K. Sadani, and B. S. Murthy, "Wavelet analysis of intermittent turbulent transport in the atmospheric surface layer over a monsoon trough region," Boundary-Layer Meteorol. 90, 217 (1999).

${ }^{61}$ J. Clyne, P. Mininni, A. Norton, and M. Rast, "Interactive desktop analysis of high resolution simulations: Application to turbulent plume dynamics and current sheet formation," New J. Phys. 9, 301 (2007). 